\title{
RECONSTRUCTION OF MATERIAL STIFFNESS PROPERTIES FROM DISPERSION RELATIONS USING GENETIC ALGORITHMS
}

\author{
V.Thierry ${ }^{1}$, W.Wu ${ }^{1}$, D.Chronopoulos ${ }^{1}$, S.Cantero Chinchilla ${ }^{2}$ \\ ${ }^{1}$ Institute for Aerospace Technology \& The Composites Research Group, The University of Nottingham, NG7 2RD, UK \\ ${ }^{2}$ Department of Mechanical Engineering, University of Bristol, Bristol, BS8 1TR, UK
}

\begin{abstract}
The widespread use of composite materials has led to the development of characteristics identification methods for composite materials. This paper presents an inverse technique based on genetic algorithms to reconstruct material stiffness properties of composites from dispersion relations of three fundamental modes of guided Lamb waves $\left(\mathrm{A}_{0}\right.$, $\mathrm{SH}_{0}$ and $\mathrm{S}_{0}$ ). The discrepancy between the mesoscopic dispersion relations computed for a textile composites and the macroscopic dispersion characteristics computed for a tentative set of elastic moduli defines the objective function. The proposed scheme is presented to obtain the elastic moduli of unidirectional carbon fibre reinforced polymer composite. The results show that the proposed method can effectively inverse the dispersion curves to get mechanical properties of composites.
\end{abstract}

Keywords: Textile composites, Guided Lamb wave, Dispersion relations, Genetic Algorithm

\section{Introduction}

Carbon fiber composite materials have been widely used in modern industries due to the characteristics of high specific strength and high specific stiffness [1,2]. Different types of damage can occur in composites structures, which potentially leads to catastrophic failure of the whole system. Therefore effective and reliable Non-Destructive Evaluation (NDE) and Structural Health Monitoring (SHM) techniques are of utter importance to develop. Various algorithms were developed for damage identification, including time-of-flight, time reversal, migration technique, tomography and phased-array beamforming $[3,4,5,6]$. All of the above algorithms require prior knowledge of elastic moduli particularly in anisotropic materials.

The traditional tests for determining the elastic properties of materials are generally expensive and time-consuming. Over years, non-destructive techniques have been done to identify the elastic constants of composite plates as these techniques are robust and quick to perform, especially guided wave technique [7,8]. The use of guided wave for material characterization yields a frequency-dependent behaviour of the waves that can be used in inverse identification procedures. Different parameters, such as wave velocity [9], group delay curves [10], wavenumber [11], and dynamic displacement response [12] are used to identify the elastic constants in the inverse process.

The solution of dispersion relations is the premise of the inversion process. Methods such as SAFE [13, 14, 15], or WFE $[16,17,18]$ using macroscale modelling are extremely efficient to compute the dispersion relations of laminates as long as the elastic moduli are known for each individual layer. The methodology presented in [18] provides a reduced formulation for WFE approach which in itself is a hybridization between periodic structure theory and finite elements. This methodology, while revealing itself to provide accurate dispersion predictions, needs much more computational resources than a macroscale model. Given that macro is superior to meso in terms of the computational time, but from the perspective of accuracy, it is opposite. Therefore, there is considerable motivation to bridge them. The approach proposed in this manuscript takes advantage of the accuracy of the methodology for computing dispersion relations in complex textile composites from [18] and the efficiency of the SAFE method to compute the dispersion characteristics of macroscale laminate models.

Different inversion procedures have been used to identify the elastic constants of composite plates [12, 19, 20, 21]. Balasubramaniam [19] was the first to employ genetic algorithms (GAs) for inverting unidirectional composite 
material elastic moduli with significant success. The advantage of GAs over other search algorithms is that GAs do not need an initial guess but rather a valid search space; GAs are also robust and avoid entrapment at local minima. Neural network techniques have also been applied in solving inverse problems [12, 20]. More recently, a Bayesian identification technique based on finite element modelling and the properties of propagating waves in multilayered structures is proposed, which overcomes the limitations of ill-conditioning and non-uniqueness associated with the conventional approaches [21]. Considering the advantages of GAs, GAs are adopted in this paper.

The paper is structured as follows. In Sec. 2, the proposed methodology is thoroughly described. In Sec. 3, the scheme is validated using a simple orthotropic plate structure. Finally, Sec. 4 provides concluding remarks.

\section{Inverse methodology based on a genetic algorithm}

In this section, the GA is used to determine the elastic moduli of a material from its dispersion relations. A schematic representation of the elastic moduli identification framework based on the GA method is shown in Fig. 1. The mesoscale model based on modified WFE approach [18]is used to calculate the mesoscale dispersion characteristics, which is regarded as the input of an inverse algorithm. The macroscale model based on SAFE method is used to calculate the iterative dispersion relations. The GWs-based identification of elastic properties is realized through the minimization of an objective function, which is built on the relative discrepancies between the dispersion characteristics computed using the mesoscale model and the SAFE method for a tentative set of elastic moduli at the macroscale. The GA procedure iteratively updates the sets of elastic moduli in the SAFE formulation in order to minimise the objective function as presented in [22]. The iteration terminates when the stopping-conditions are fulfilled; these can be when the value of the objective function for a tentative set of elastic moduli overcomes a threshold or after completing a predefined number of iterations.

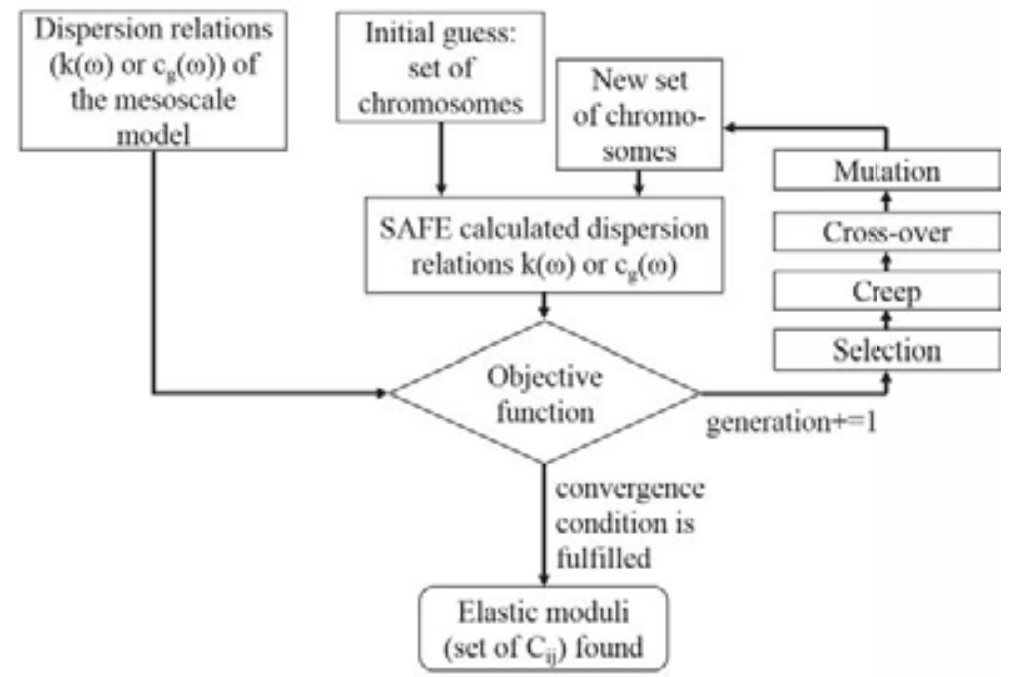

Figure 1: Schematic representation of the elastic moduli $\left(C_{i j}\right)$ identification framework based on a genetic algorithm. The starting point for the optimization process are the nine statically determined mechanical moduli for the textile composite. The mesoscale wave propagation properties computed through a modified WFE scheme are compared to the macroscale SAFE estimations incorporating the mechanical moduli. The moduli are updated iteratively until the mesoscale and macroscale wave propagation properties converge.

In this study, a chromosome is a set of nine elastic moduli (also called $C_{i j}$ ) describing the stiffness matrix of the homogenised orthotropic material. Each individual modulus is a gene. A set of multiple chromosomes is called a population, as depicted in Fig. 2.

\subsection{Initialisation of the homogenisation process}

Although it is not necessary for attaining convergence, using an initial guess allows for speeding up the process (that is given that the initial guess is not far from the solution). Since the material is modelled to obtain its mesoscale 


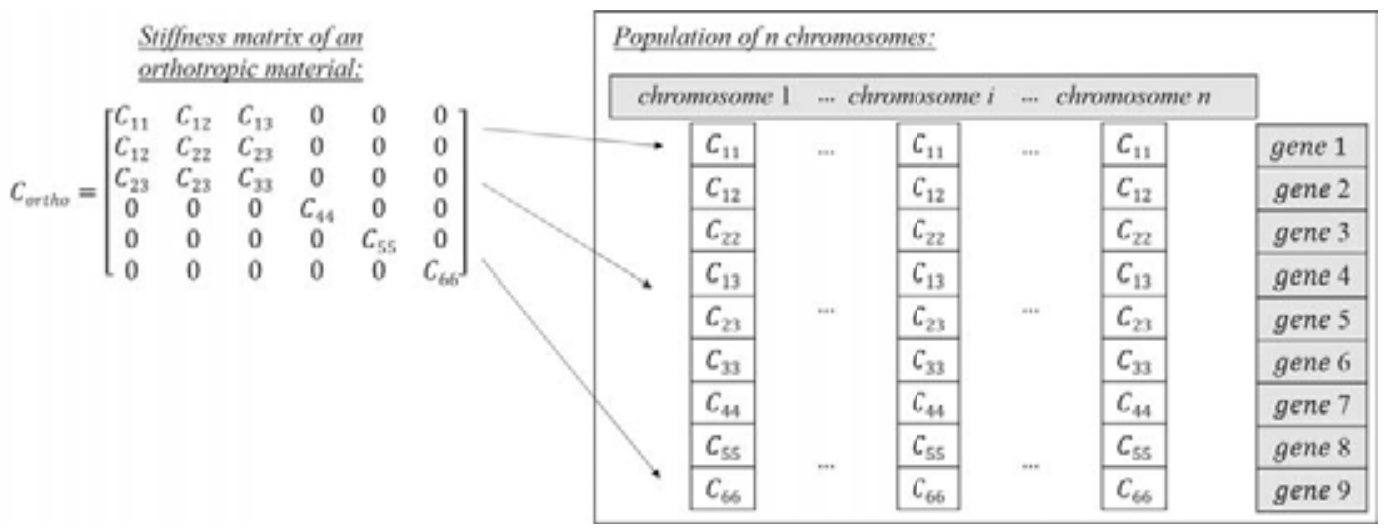

Figure 2: Chromosomes incorporating the elastic moduli of an orthotropic textile layer. Multilayer structures can be considered with the size of chromosomes varying accordingly. Nine idependent elastic moduli are included for each layer. These moduli are injected within a macroscale SAFE calculation and results are compared against the mesoscale derived wave dispersion properties.

dispersion characteristics, it is convenient to use its effective elastic moduli obtained by static virtual testing (method detailed in $[23,24,18]$ ) as initial guess but not necessary. A small set of chromosomes of the initial population is set equal to the initial guess while the rest of the population is randomly generated from a small neighborly interval whose median value is the initial guess.

\subsection{Objective function}

The objective function defines the discrepancy between the mesoscopic dispersion relations computed for a textile composite and the macroscopic dispersion characteristics computed for a tentative set of elastic moduli. It is noted that any property directly related to the dispersion characteristics of the structure (i.e. the computed wavelength, wavenumber of wave speed) can be employed within the objective function in Eq. (1) with such correlated quantities being expected to provide the same effective properties. The identification of the optimal set of elastic moduli is realised through the minimisation of this objective function. The mesoscale dispersion relations are input to the GA procedure and are not updated while the macroscale dispersion curves are computed iteratively using the SAFE method for each updated set of elastic moduli. Different types of dispersion characteristics can be used for comparison: the wavenumber or the group velocity displayed in Fig. 3 are two possibilities. While the group velocity values are traditionally used [25, 26, 27] for GA-based homogenisation methods (for the simple reason that this parameter is straightforward to obtain experimentally), the wavenumber parameter is also a good candidate. In this study, the wavenumber is used to explore more possibilities.

The error function to be minimised is the Mean of the Relative Error (MRE) for each point of an individual dispersion curve and is written as follows:

$$
\operatorname{MRE}(\theta)=\sum_{i=1}^{n}\left|\frac{\lambda_{i}^{\text {meso }}(\theta)-\lambda_{i}^{\text {macro }}(\theta)}{\lambda_{i}^{\text {meso }}(\theta)}\right| / n .
$$

with $\lambda$ the dispersion parameter which is hereby taken as the computed wavelength (equivalently the phase velocity) of the considered wave mode. Moreover $\theta$ the angle of propagation of consideration and $n$ the number of compared data points.

The relative error function is computed separately for the three fundamental modes $\left(\mathrm{S}_{0}, \mathrm{SH}_{0}\right.$ and $\left.\mathrm{A}_{0}\right)$ and in a few directions of propagation. The objective function is set to the maximum of any of these values, thus minimising all modes simultaneously. All modes have to be considered as it would be useless to obtain a set of moduli that reconstructs one mode only considering that results will be employed within wave interaction with damage modelling or multiple ray reflection modelling where wave conversion phenomena are predominant. Using the sum of all relative errors has been considered but revealed itself to slow down the optimisation process. It is noted that while the formulation is globally applicable to frequency ranges including higher order modes, this study focuses on matching 


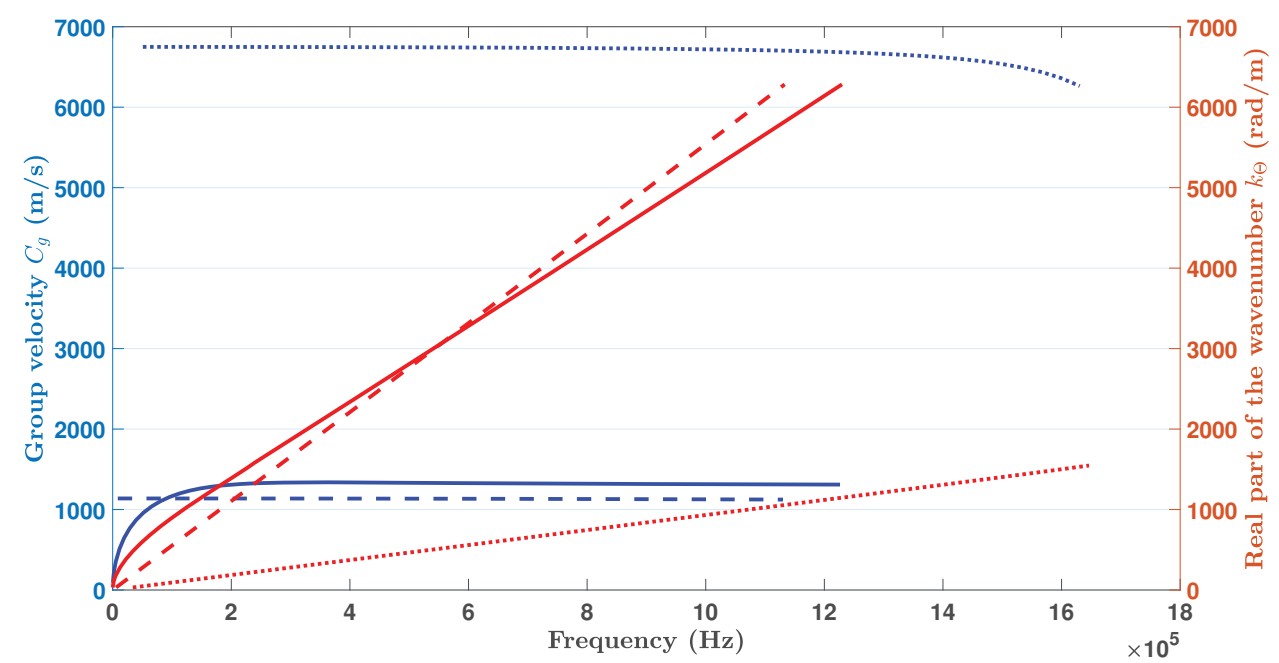

Figure 3: Dispersion relations (group velocity plotted in blue and wavenumber in red) of the three first modes in the $x$-direction of propagation for a plate constituted of an orthotropic material. Comparisons on the sensitivity of both parameters are provided in Sec. 3 to determine which is best for this application. (-) $\mathrm{A}_{0},(--) \mathrm{SH}_{0}$, (..) $\mathrm{S}_{0}$.

the dispersion characteristics within the range where only fundamental Lamb wave modes propagate, since this is the range typically employed for damage identification.

\subsection{Selection and genetic operators}

Selection of the portion of existing population to breed. The fitness function is directly derived from the objective function and determines the likeliness of a chromosome to be selected for the next generation. The selection process can be visualised as a roulette wheel in which each chromosome covers an area of the wheel proportional to its probability to be selected. The chromosomes for the next generation are selected one by one by turning the wheel. All generations population contain an equal number of chromosomes thus a chromosome with a high probability of selection might be selected more than once to be passed on to the next generation.

Creeping operation. A creeping operation is performed on each new candidate solution. A threshold is established and a random number is generated. If the random number exceeds the threshold then the considered set of elastic moduli is randomly scaled in the range of $\left[1-\delta_{\text {creep }}, 1+\delta_{\text {creep }}\right], \delta_{\text {creep }}$ being the creep amount. Using this approach also allows for a search outside the search space.

Single-point crossover of parent chromosomes. Some of the selected chromosomes mate through the crossover process to create new offspring whose genes are a combination of its parent's genes. The crossover rate defines the number of parent chromosomes to be selected for cross-over. Two parent chromosomes are 'cut' at a single random crossover point and their genes are interchanged.

Mutation of parent chromosomes. Finally some of the chromosomes are mutated to avoid stagnation of the solution to a local minima. The mutation rate defines the number of genes to be mutated and their positions across the chromosomes are selected randomly. The genes selected for mutations are each replaced by a newly generated one.

\section{Case studies}

To show the applicability of the method, it is firstly performed on a simulated orthotropic plate whose theoretical elastic moduli are known. Its dispersion characteristics are computed using the WFE method similarly to the way it would for a textile composite. In the following subsections, the elastic moduli are approximated using two different approaches to validate and compare them. The approach called 'brute-force' with the ambitious task of reconstructing all nine parameters at once is adopted here. 


\subsection{Brute-force approach}

The thickness of the plate and the density of the material are fixed $\left(0.5 \mathrm{~mm}\right.$ and $\left.3212 \mathrm{~kg} / \mathrm{m}^{3}\right)$ as known and the nine elastic moduli presented in Table1 in the 'theoretical' column are sought. These moduli represent a unidirectional carbon fibre reinforced polymer composite whose fibre arrangement makes it slightly non-transverse isotropic. Table1 shows the results obtained for five different runs of the GA and the percentage of error to the theoretical value. In order to validate the method, a random set of elastic moduli was used as initial guess for each run. The crossover and mutation rates are both set to 0.1 , the creep amount is set to 0.3 and the generated population size is 50 chromosomes. The algorithm is launched considering the wavenumber dispersion relations for five runs. Parallel computing is performed on a 4 cores and $8 \mathrm{~Gb}$ RAM system and the algorithm is coded in Python language.

\begin{tabular}{|l|l|l|l|l|l|l|}
\hline & Theoretical & $\mathbf{\# 1}$ & $\mathbf{\# 2}$ & $\mathbf{\# 3}$ & $\mathbf{\# 4}$ & $\mathbf{\# 5}$ \\
\hline$C_{11}(\mathrm{GPa})$ & 147.36 & 146.18 & 147.61 & 146.93 & 147.39 & 148.12 \\
error $(\%)$ & & 0.80 & 0.18 & 0.29 & 0.02 & 0.52 \\
\hline$C_{12}(\mathrm{GPa})$ & 3.43 & 2.46 & 2.93 & 3.85 & 3.63 & 8.64 \\
error $(\%)$ & & 28.23 & 14.49 & 12.18 & 5.68 & 151.65 \\
\hline$C_{22}(\mathrm{GPa})$ & 9.51 & 8.68 & 8.21 & 8.34 & 9.31 & 10.11 \\
error $(\%)$ & & 8.71 & 13.70 & 12.29 & 2.15 & 6.30 \\
\hline$C_{13}(\mathrm{GPa})$ & 3.47 & 1.11 & 0.73 & 1.18 & 1.77 & 5.88 \\
error $(\%)$ & & 67.84 & 78.84 & 65.85 & 48.83 & 69.59 \\
\hline$C_{23}(\mathrm{GPa})$ & 4.02 & 1.49 & 1.31 & 1.01 & 1.89 & 4.87 \\
error $(\%)$ & & 63.04 & 67.49 & 74.94 & 52.98 & 21.17 \\
\hline$C_{33}(\mathrm{GPa})$ & 11.66 & 5.77 & 5.19 & 5.53 & 4.80 & 12.89 \\
error $(\%)$ & & 50.52 & 55.49 & 52.54 & 58.85 & 10.54 \\
\hline$C_{44}(\mathrm{GPa})$ & 3.19 & 3.18 & 3.36 & 3.30 & 3.20 & 3.21 \\
error $(\%)$ & & 0.20 & 5.44 & 3.44 & 0.31 & 0.56 \\
\hline$C_{55}(\mathrm{GPa})$ & 5.58 & 5.60 & 5.69 & 5.72 & 5.48 & 5.59 \\
error $(\%)$ & & 0.35 & 1.99 & 2.52 & 1.88 & 0.22 \\
\hline$C_{66}(\mathrm{GPa})$ & 4.16 & 4.13 & 4.14 & 4.10 & 4.13 & 4.09 \\
error $(\%)$ & & 0.72 & 0.41 & 1.51 & 0.57 & 1.54 \\
\hline Generations & - & 103 & 118 & 286 & 222 & 222 \\
\hline Time $(\mathrm{h})$ & - & 25 & 30 & 59 & 49 & 46 \\
\hline
\end{tabular}

Table 1: Results of the GA for five independent runs for the reconstruction of the elastic moduli of an orthotropic material whose real moduli are displayed on the left-hand side, when $k(\omega)$ is considered.

The results are displayed for the wavenumber approach in Table1. It can be observed that this methodology, while providing a realistic order of magnitude for the elastic moduli, yields strong relative error for many of them $(>10 \%)$. Some elastic moduli such as $C_{11}$ and $C_{66}$ however, are in all five runs approximated with a very low relative error $(<2 \%)$, while $C_{44}$ and $C_{55}$ are approximated with a tolerable error $(<6 \%)$. One can conclude that these parameters are extremely sensible in comparison to the others.

Trying to optimise nine coefficients at once is a very ambitious task and the convergence is obtained after many generations (at least 100) as seen in Fig. 4.a, and thus is computationally taxiing. Moreover, only two elastic moduli are reconstructed with good accuracy and two more with a relatively low error. One can conclude that this approach is neither time efficient, nor yields accurate results. However, the objective function value is smaller than $1 \%$ at convergence for each of the runs. This indicates that even though the correct solution was not found, at least a solution does exist. Therefore, there is a considerable motivation to employ the sensitivity of each individual mode to the different moduli to optimise a reduced number of parameters at once in the future work. 


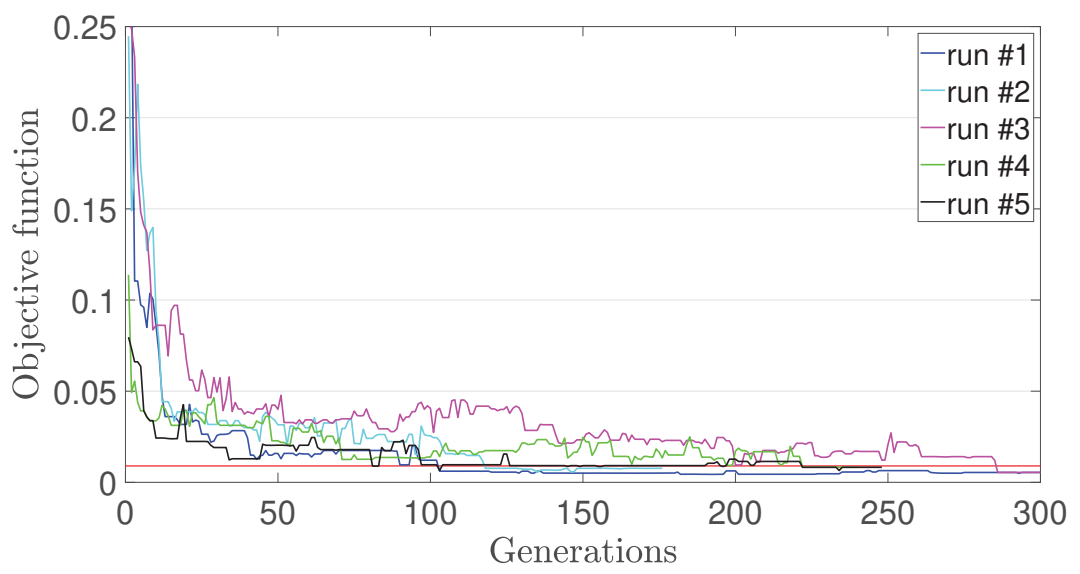

Figure 4: Value of the objective function as a function of the generation for the five GA independent runs for the reconstruction of the elastic moduli of the orthotropic material. $k(\omega)$ is considered. Convergence is observed for all runs after 290 generations. 100 generations were needed for at least one run to converge. The horizontal red line is plotted at $1 \%$ of error.

\section{Conclusions}

The GA is presented to reconstruct material stiffness properties of composites based on dispersion characteristics of Lamb wave in this paper, which combines the advantages of WFE and SAFE. Case study has been selected to illustrate the strengths of proposed approach. While the brute-force approach showed that the dispersion relations have a very low sensitivity to some of the moduli if considered altogether. A sensitivity study is therefore hereby performed in order to uncover the effect of each parameters to be optimised on the dispersion relations in the future.

\section{Acknowledgments}

- This work is funded by the INNOVATIVE doctoral programme. The INNOVATIVE programme is partially funded by the Marie Curie Initial Training Networks (ITN) action (project number 665468) and partially by the Institute for Aerospace Technology (IAT) at the University of Nottingham. This project has also received funding from the European Union's Horizon 2020 research and innovation programme under the Marie SkłodowskaCurie grant agreement No. 859957.

- We are grateful for access to the University of Nottingham's Augusta HPC service. 


\section{References}

[1] R. Bai, J. Guo, Z. Lei, D. Liu, Y. Ma, and C. Yan, "Compression after impact behavior of composite foam-core sandwich panels," Composite Structures, vol. 225, p. 111181, 2019.

[2] R. Bai, Z. Lei, X. Wei, W. Tao, and C. Yan, "Numerical and experimental study of dynamic buckling behavior of a j-stiffened composite panel under in-plane shear," Composite Structures, vol. 166, pp. 96-103, 2017.

[3] S. Cantero-Chinchilla, J. Chiachío, M. Chiachío, D. Chronopoulos, and A. Jones, "A robust bayesian methodology for damage localization in plate-like structures using ultrasonic guided-waves," Mechanical Systems and Signal Processing, vol. 122, pp. 192-205, 2019.

[4] P. Malinowski, T. Wandowski, I. Trendafilova, and W. Ostachowicz, "A phased array-based method for damage detection and localization in thin plates," Structural Health Monitoring, vol. 8, no. 1, pp. 5-15, 2009.

[5] Z. Su and L. Ye, "Selective generation of lamb wave modes and their propagation characteristics in defective composite laminates," Proceedings of the Institution of Mechanical Engineers, Part L: Journal of Materials: Design and Applications, vol. 218, no. 2, pp. 95-110, 2004.

[6] H. W. Park, S. B. Kim, and H. Sohn, "Understanding a time reversal process in lamb wave propagation," Wave Motion, vol. 46, no. 7, pp. 451-467, 2009.

[7] L. Pagnotta, "Recent progress in identification methods for the elastic characterization of materials," International Journal of mechanics, vol. 2, no. 4, pp. 129-140, 2008.

[8] J. H. Tam, Z. C. Ong, Z. Ismail, B. C. Ang, and S. Y. Khoo, "Identification of material properties of composite materials using nondestructive vibrational evaluation approaches: A review," Mechanics of Advanced Materials and Structures, vol. 24, no. 12, pp. 971-986, 2017.

[9] J. Vishnuvardhan, C. Krishnamurthy, and K. Balasubramaniam, "Blind inversion method using lamb waves for the complete elastic property characterization of anisotropic plates," The Journal of the Acoustical Society of America, vol. 125, no. 2, pp. 761-771, 2009.

[10] A. Marzani and L. De Marchi, "Characterization of the elastic moduli in composite plates via dispersive guided waves data and genetic algorithms," Journal of intelligent material systems and structures, vol. 24, no. 17, pp. 2135-2147, 2013.

[11] A. A. Eremin, E. Glushkov, N. Glushkova, and R. Lammering, "Evaluation of effective elastic properties of layered composite fiber-reinforced plastic plates by piezoelectrically induced guided waves and laser doppler vibrometry," Composite Structures, vol. 125, pp. 449-458, 2015.

[12] G. Liu, K. Lam, and X. Han, "Determination of elastic constants of anisotropic laminated plates using elastic waves and a progressive neural network," Journal of Sound and Vibration, vol. 252, no. 2, pp. 239-259, 2002.

[13] I. Bartoli, A. Marzani, F. Lanza di Scalea, and E. Viola, "Modeling wave propagation in damped waveguides of arbitrary cross-section," Journal of Sound and Vibration, vol. 295, no. 3-5, pp. 685-707, 2006.

[14] A. Marzani, E. Viola, I. Bartoli, F. L. Di Scalea, and P. Rizzo, "A semi-analytical finite element formulation for modeling stress wave propagation in axisymmetric damped waveguides," Journal of Sound and Vibration, vol. 318, no. 3, pp. 488-505, 2008.

[15] K.-L. Nguyen, F. Treyssede, and C. Hazard, "Numerical modeling of three-dimensional open elastic waveguides combining semi-analytical finite element and perfectly matched layer methods," Journal of Sound and Vibration, vol. 344, pp. 158-178, 2015.

[16] D. Mead, "Wave propagation in continuous periodic structures: research contributions from southampton, 1964-1995," Journal of sound and vibration, vol. 190, no. 3, pp. 495-524, 1996.

[17] B. R. Mace, D. Duhamel, M. J. Brennan, and L. Hinke, "Finite element prediction of wave motion in structural waveguides," The Journal of the Acoustical Society of America, vol. 117, no. 5, pp. 2835-2843, 2005.

[18] V. Thierry, L. Brown, and D. Chronopoulos, "Multi-scale wave propagation modelling for two-dimensional periodic textile composites," Composites Part B: Engineering, vol. 150, pp. 144-156, 2018.

[19] K. Balasubramaniam and N. S. Rao, "Inversion of composite material elastic constants from ultrasonic bulk wave phase velocity data using genetic algorithms," Composites Part B: Engineering, vol. 29, no. 2, pp. 171-180, 1998.

[20] G. Liu, W. Ma, and X. Han, "An inverse procedure for determination of material constants of composite laminates using elastic waves," Computer Methods in Applied Mechanics and Engineering, vol. 191, no. 33, pp. 3543-3554, 2002.

[21] W.-J. Yan, D. Chronopoulos, S. Cantero-Chinchilla, K.-V. Yuen, and C. Papadimitriou, "A fast bayesian inference scheme for identification of local structural properties of layered composites based on wave and finite element-assisted metamodeling strategy and ultrasound measurements," Mechanical Systems and Signal Processing, vol. 143, p. 106802, 2020.

[22] A. Marzani and L. De Marchi, "Characterization of the elastic moduli in composite plates via dispersive guided waves data and genetic algorithms," Journal of Intelligent Material Systems and Structures, vol. 24, no. 17, pp. 2135-2147, 2012.

[23] S. Li and A. Wongsto, "Unit cells for micromechanical analyses of particle-reinforced composites," Mechanics of Materials, vol. 36, no. 7, pp. 543-572, 2004.

[24] S. Li and E. Sitnikova, 1.18 An Excursion into Representative Volume Elements and Unit Cells, pp. 451-489. Oxford: Elsevier, 2018.

[25] J. Vishnuvardhan, C. V. Krishnamurthy, and K. Balasubramaniam, "Genetic algorithm based reconstruction of the elastic moduli of orthotropic plates using an ultrasonic guided wave single-transmitter-multiple-receiver shm array," Smart Materials and Structures, vol. 16, no. 5, p. $1639,2007$.

[26] M. Sale, P. Rizzo, and A. Marzani, "Semi-analytical formulation for the guided waves-based reconstruction of elastic moduli," Mechanical Systems and Signal Processing, vol. 25, no. 6, pp. 2241-2256, 2011.

[27] A. A. Eremin, E. V. Glushkov, N. V. Glushkova, and R. Lammering, "Evaluation of effective elastic properties of layered composite fiberreinforced plastic plates by piezoelectrically induced guided waves and laser doppler vibrometry," Composite Structures, vol. 125, pp. 449$458,2015$. 